\title{
QUANTIFICATION OF CONTRAST \\ RECOGNIZABILITY IN SEQUENTIAL EPIRETINAL MEMBRANE REMOVAL AND INTERNAL LIMITING MEMBRANE PEELING IN TRYPAN BLUE-ASSISTED MACULAR SURGERY
}

\author{
PAUL B. HENRICH, MD, $*$ SIEGFRIED G. PRIGLINGER, MD,$\dagger+$ CHRISTOS HARITOGLOU, MD, $\dagger$ \\ RICARDA G. SCHUMANN, MD, $\dagger$ RUPERT W. STRAUSS, MD, $\ddagger \S$ ULRIKE SCHNEIDER, MD,* \\ TATJANA JOSIFOVA, MD,* PHILIPPE C. CATTIN, MSII
}

Purpose: To evaluate the selectivity and strength of intraoperative trypan blue staining during removal of epiretinal membranes (ERMs) and the internal limiting membrane.

Methods: Based on intraoperative videos, 51 consecutive chromovitrectomies in 51 patients with macular holes, macular pucker, vitreomacular traction syndromes, or persistent macular edema were retrospectively studied. Fifteen subjects underwent trypan blue, 14 indocyanine green, and 22 brilliant blue G chromovitrectomy. The main outcome measure was the color contrast between stained internal limiting membrane or ERM and the underlying unstained tissue by means of objective, quantitative, semiautomated chromaticity difference measurements.

Results: Trypan blue stains both ERM and the internal limiting membrane (average chromaticity scores 8.51 and 7.09 , respectively; $P=0.48$ ). Internal limiting membrane chromaticity scores were similar for trypan blue $(7.09)$ and brilliant blue $\mathrm{G}(6.81 ; P=0.71)$ but clearly higher for indocyanine green $\left(15.81 ; P=2.45 \times 10^{-5}\right)$.

Conclusion: Under the premises of our study, trypan blue stains both ERM and the internal limiting membrane. Trypan blue's staining capacity of the internal limiting membrane is similar to that of brilliant blue G but significantly inferior compared with indocyanine green. Trypan blue, thus, represents a useful vital dye for chromovitrectomy, particularly in the presence of ERM, where it allows a sequential approach.

RETINA 33:818-824, 2013

A nteroposterior and tangential posterior pole vitreoretinal traction is causal in the formation of a multitude of macular pathologies, including macular holes, vitreomacular traction syndrome, persistent macular

From the *Department of Ophthalmology, University Hospital Basel, Basel, Switzerland; †University Eye Hospital of the LudwigMaximilians University, Munich, Germany; $\ddagger$ Department of Ophthalmology and Optometry, Linz General Hospital, Linz, Austria; §Department of Ophthalmology, Medical University Graz, Graz, Austria; and IIMedical Image Analysis Center, University of Basel, Basel, Switzerland.

The authors declare no conflict of interest.

Reprint requests: Paul B. Henrich, MD, Department of Ophthalmology, University of Basel Eye Hospital, PO Box, CH-4012 Basel, Switzerland; e-mail: Henrichp@uhbs.ch edema, and macular pucker. ${ }^{1}$ Common clinical characteristics of these conditions include visual loss and metamorphopsias.

A fundamental approach to diseases based on vitreoretinal traction has become attainable with the introduction of pars plana vitrectomy. ${ }^{2}$ However, mechanical vitrectomy is often incomplete. Even if epiretinal membranes (ERMs) are removed thoroughly, residual cortical vitreous attached to the internal limiting membrane (ILM) may account for sustained traction. ${ }^{1}$ Although originally regarded as an unintentional side effect of ERM removal, ${ }^{3}$ peeling of the ILM has been demonstrated to improve anatomical outcomes through a more complete release of tractional forces 
and inhibition of reproliferation of fibrous astrocytes, ${ }^{4}$ resulting in higher rates of macular hole closure and improved visual outcomes. ${ }^{5-8}$

Both the pellucid and highly delicate ERM and ILM may be technically difficult to detach from the substrate. As a result, damage to the underlying retina in the form of intraretinal hemorrhages, central retinal breaks, and functional retinal injury may ensue. $^{9-11}$ The completeness of ILM removal is also difficult to ensure. To improve safety and completeness of ERM and ILM removal, many surgeons rely on the application of vital dyes to increase the visibility of the target tissue, an approach frequently referred to as chromovitrectomy. ${ }^{12}$ If both ERM and the ILM are intended to be removed, one or several dyes may be used sequentially to differentiate the different tissue layers. A great deal of disarray exists, however, regarding which biostain to choose for ILM removal and for sequential macular surgery. A suitable vital dye for ILM peeling would combine strong ILM staining with a favorable safety profile and approval status. For sequential interventions, a strong additional affinity to ERM would be desirable. Trypan blue (TB) has been proposed for ERM staining, but uncertainty revolves around its affinity to the ILM. ${ }^{13}$

\section{Materials and Methods}

In a retrospective clinical case series, we analyzed 51 consecutive chromovitrectomy interventions in 51 patients with macular holes, macular pucker, persistent macular edema, and vitreomacular traction syndrome. Twenty-four right eyes and 27 left eyes were included. Patient age ranged from 47 years to 89 years (median 74.1 years). Twenty-four patients were women, 27 were men. Four patients were phakic and 27 were pseudophakic, and in 20 interventions, a phacoemulsification and an insertion of a posterior chamber intraocular lens into the capsular bag preceded chromovitrectomy. Exclusion criteria included patients younger than 18 years, technically poor video quality, and previous chromovitrectomies within the preceding 6 months.

All patients underwent routine 23-gauge vitrectomy under local anesthesia, performed by 4 surgeons (P.B.H., S.G.P., T.J., and U.S.) at 2 centers (Basel, Switzerland; Linz, Austria) using the OS3 vitrectomy system (Oertli, Berneck, Switzerland) in combination with a Photon II light source (Synergetics, O'Fallon, MO).

The choice of the vital dye was based on the preference of the individual surgeon. After complete posterior vitreous detachment and vitrectomy, one of three vital dyes was injected into the vitreous cavity:

- Trypan blue $0.15 \%$ was taken from ready-to-use 0.5 -mL syringes stored at $4^{\circ} \mathrm{C}$ (MembraneBlue; DORC International BV, Zuidland, the Netherlands). The complete content of the syringe was injected under balanced salt solution (BSS) and removed after 10 seconds.

- Brilliant blue G (BBG) $0.025 \%$ from ready-to-use $0.5-\mathrm{mL}$ vials stored at room temperature (Brilliant Peel; Geuder AG, Heidelberg, Germany) was instilled completely under BSS with immediate washout.

- Indocyanine green (ICG) $1.25 \%$ was prepared as an isoosmolar solution stored at room temperature and a volume of $0.5 \mathrm{~mL}$ was applied intravitreally with clearance occurring after 60 seconds (ICG Pulsion; Pulsion Medical Systems AG, Munich, Germany).

Membrane removal recordings were performed as described previously. ${ }^{14}$ Briefly, all interventions were recorded using a Medlife Trio Digital Camera in connection with a Medlife Mind Stream Digital Recorder and an Opmi Visu 200 Ophthalmic Microscope (all Carl Zeiss Meditec, Jena, Germany). Exposure and calibration alignments were routinely performed.

The video sequences were viewed and analyzed postoperatively using a custom-made software toolprogrammed in MATLAB (Version R2007b, The MathWorks, Inc., Natick, MA) as described earlier. ${ }^{14}$ Frames displaying good image quality were selected, and regions of interest (ROIs) representing maximum contrast were signaled within the temporal vascular arcades under the supervision of a vitreoretinal surgeon (P.B.H.). For classification of ERM versus ILM, all videos were reviewed with the surgeon. Allocation of a frame to either the ERM or ILM group is, thus, a representation of the surgeon's clinical intraoperative judgment, mainly based on morphologic appearance and order of peeling events. Histologic confirmation was not available because of the retrospective nature of the study. For contrast calculation, two distinct methods were used: In the single image method, one ROI was selected in an area with maximally stained ILM and compared with another adjacent ROI of similar dimensions in the same image in an area where the ILM had already been removed during the course of the procedure. In the multiple image method, an area with maximum staining was selected and the same ROI was assessed at different points in time, before and after ILM removal. In total, 317 measurements were performed: 84 based on the single image method and 233 measurements using the multiple image method. 
Equivalence of both methods had been described previously, ${ }^{14}$ allowing pooling of data.

To quantitatively compare the perceived color contrast of the selected ROIs, the software first calculated the average color within each ROI. The perceived color contrast in the 2 regions was then determined by projecting the 2 averaged colors into the CIE $1976\left(\mathrm{~L}^{*}, \mathrm{a}^{*}, \mathrm{~b}^{*}\right)$ color space. In the CIELAB color space, changes of the same visual importance result in the same color space distance (Euclidean distance). The Euclidean distance is therefore a direct measure for perceivable visual color difference. ${ }^{14}$ To assure independence of lighting variations, the Euclidean distance was only calculated over the chromaticity components $\mathrm{a}^{*}$ and $\mathrm{b}^{*}$, omitting the lightness $\mathrm{L}^{*}$.

Statistical analysis was performed with the software package R Version 2.11.1. ${ }^{15}$ Normality of the distributions was assured with the Kolmogorov-Smirnov test, and a $t$-test was used to assess significance. To compare subgroup mean values, one-way analyses of variance were performed. A $P$ value $<0.05$ was considered significant.

At all times, the tenets of the Declaration of Helsinki were observed. The protocol was approved by the local ethics committee.

\section{Results}

A clinically useful staining, sufficient for ERM and/ or ILM removal, was observed in all interventions with the dye of first choice.

Chromaticity diagram analysis of patients operated with $\mathrm{TB}$ showed slightly higher CIELAB distances between ERM and the underlying unstained tissue compared with the ILM and subjacent unstained retina, although a $t$-test showed no statistical difference between both groups. Average CIELAB distance was 8.51 for the ERM group and 7.09 for the ILM group, $P=0.48$ (Figure 1).

Comparison of the different vital dyes' affinities to the ILM showed similar values for TB compared with BBG but significantly inferior results when matched with ICG. Average CIELAB distance was 7.09 for the TB group, as against 6.81 for the BBG group $(P=0.71)$ and 15.81 for the ICG group $\left(P=2.45 \times 10^{-5}\right)$ (Figure 2).

Chromaticity data for all subgroups revealed a Gaussian distribution. Comparison of disease subgroup mean values revealed $P$ values of 0.12 for subjects operated with TB and 0.52 and 0.98 for eyes operated with brilliant blue G and ICG, respectively. As all results were statistically nonsignificant, no further comparison of subgroups was needed. In particular,

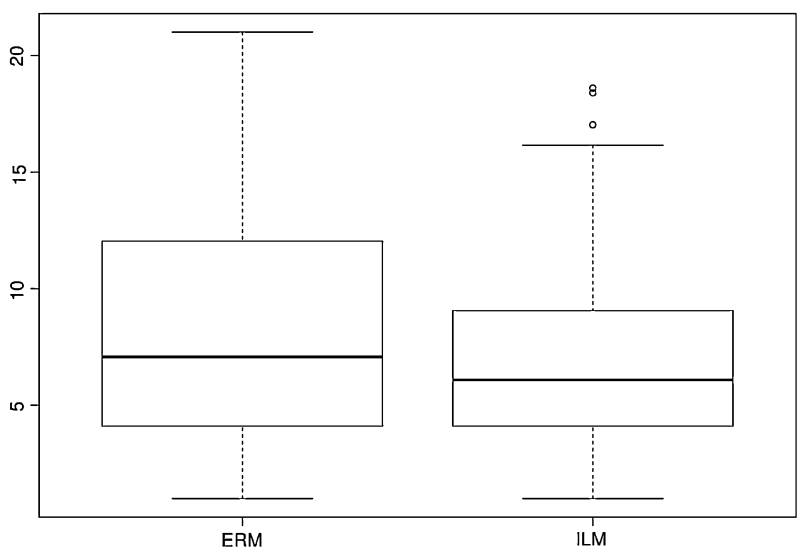

Fig. 1. Trypan blue stains ERM slightly better than the ILM, but statistical significance is not reached $(P=0.48)$. The ordinate displays the CIELAB score (Euclidean distance). The upper and lower margins of the boxes in this standard box-and-whisker diagram represent the 25th and the 75th percentile and the central line inside the box the 50th percentile (median). The whiskers mark the minimum and the maximum, with some outliers plotted as small circles.

pairwise $t$-tests and adjustment of $P$ values for multiple comparison using the Holmes correction method were not performed because of nonsignificance.

\section{Discussion}

Intraoperative staining of the ILM contributes to making its removal safer, easier, and more complete. ${ }^{5,8,16}$ The search for a truly adequate substance for this purpose is ongoing, however, because available compounds fail to combine maximum intraoperative utility with a satisfactory safety profile:

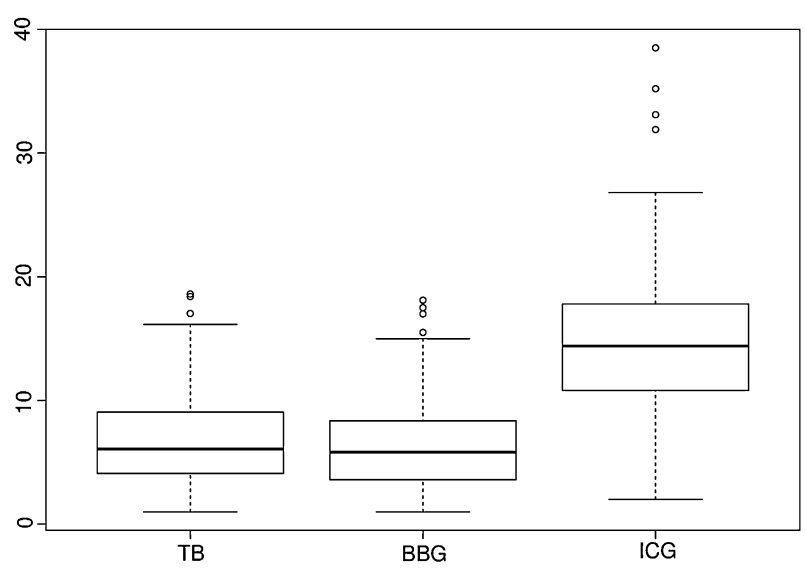

Fig. 2. Trypan blue stains the ILM similarly well as BBG $(P=0.71)$ but significantly more weakly than ICG $\left(P=2.45 \times 10^{-5}\right)$. The ordinate displays the CIELAB score (Euclidean distance). The upper and lower margins of the boxes in this standard box-and-whisker diagram represent the 25 th and the 75 th percentile and the central line inside the box the 50th percentile (median). The whiskers mark the minimum and the maximum, with some outliers plotted as small circles. 
- Indocyanine green has been used as an adjunct for chromovitrectomy for almost a decade ${ }^{17}$ as it selectively stains the ILM. ${ }^{18}$ Its staining characteristics are excellent, ${ }^{19}$ but numerous reports on toxic side effects have generated a persistent controversy. ${ }^{20-24}$ Indocyanine green is approved for intravenous use, while its intravitreal application represents an off-label use.

- Brilliant blue $\mathrm{G}$ has recently been approved for intravitreal use in the European Union. Although in vitro toxicity has recently been reported, ${ }^{25,26}$ initial clinical reports demonstrate favorable anatomical and functional results with no apparent toxicity. ${ }^{19,27,28}$ However, while BBG also selectively stains the $\mathrm{ILM},{ }^{27}$ staining intensity has been reported to be significantly inferior to that of ICG. ${ }^{14,19}$

- After its recent Food and Drug Administration's approval, TB is now also approved for intravitreal use in both Europe and the United States. Trypan blue had been used for staining of the anterior capsule in cataract surgery for over a decade before. ${ }^{29}$ It displays outstanding staining of ERMs, ${ }^{30,31}$ while evidence on its affinity to the ILM is controversial. ${ }^{13,30,32,33}$ Trypan blue is generally recommended for the removal of epiretinal tissue rather than for ILM peeling itself, ${ }^{34}$ although several clinical studies suggest that TB may be more useful for ILM removal than customarily conceived. ${ }^{31-33,35-37}$ Retinal toxicity has been occasionally reported from in vitro experiments ${ }^{38}$ and in vivo studies, ${ }^{39,40}$ while other authors found no indications for in vivo toxicity. ${ }^{16,37,41-43}$

The presence of ERM overlying the ILM requires a sequential approach. To this end, the use of multiple vital stains during the same operation has been proposed, ${ }^{44,45}$ where the first dye intends to stain ERM, while a second dye is injected after the removal of ERM to stain the ILM. Alternatively, we have demonstrated that sequential peeling may be attained with the use of a single ILM-specific vital dye. In this case, circumscribed ERM will be identified as unstained areas surrounded by tinged ILM. After ERM removal, the same vital dye is injected a second time, now having access to the exposed ILM. ${ }^{46}$

Although chromaticity measurements performed in this study were based on digital video clips, the transferal of measurement results into the CIELAB color space has been recognized to accurately reproduce human contrast discrimination capacity. ${ }^{14}$ CIELAB scores in this study, thus, are a measure of how well the surgeon will recognize the stained structures intraoperatively.

While affinity of TB to ERM is uncontroversial, our finding that it stains the ILM equally well is in accord with those who have previously advocated TB for ILM removal $^{33,36,37}$ but is at variance with others who oppose a TB affinity to acellular structures, including the ILM. ${ }^{30,47}$ Whether TB stains the ILM directly or whether fine cellular debris overspreading the ILM is responsible $e^{13,47}$ is to be elucidated in the future.

Although TB is shown to stain the ILM as well as BBG in this study, somewhat more adverse safety profile of TB needs to be pondered, when considering it as an alternative to BBG in chromovitrectomy. Safety concerns may be, at least in part, explained by considerably higher concentration of TB compared with BBG. The concentration of a vital dye necessary to allow useful intraoperative staining depends on the proportion of the substance's spectral absorption maximum located within the spectrum of visible light. ${ }^{48}$ While there is large overlap between the spectrum of visible light and absorption of BBG, including the absorption maximum, ${ }^{49}$ the TB absorption spectrum is gently displaced toward longer wavelengths, ${ }^{50}$ requiring higher concentrations for equal staining results. Absorption of ICG spectrum is predominantly located in the near infrared spectrum, ${ }^{48}$ and its outstanding staining characteristics are reached at the expense of high dye concentrations and the notable toxicity profile.

In the light of the correlation between dye concentration and intraocular toxicity, the use of a mixture of BBG and $\mathrm{TB}$ at low concentrations might improve results over the use of the individual agents. A combination vital dye containing $\mathrm{BBG}$ at $0.025 \%$ and $\mathrm{TB}$ at $0.15 \%$ has recently been launched (Membrane Blue Dual; DORC International BV). Initial practical experiences with this compound are encouraging in our hands, and first in vitro studies report good safety. ${ }^{26}$ Clinical or experimental reports are not yet available at this time.

Trypan blue distinguishes itself from all other available intravitreal dyes by staining both ERM and ILM. Trypan blue can, thus, be seen as an interesting alternative to BBG for the removal of both ERM and ILM (double peeling). Trypan blue offers the surgeon the advantage that all tissues to be removed can be stained with the same vital dye.

The patient population used in this study consists of a group of four related diseases, whose configurations might influence staining characteristics. However, subgroup analyses of variance revealed that staining was not affected by the underlying pathology in any of the vital dye groups with $P$ values of 0.12 for subjects operated with TB and 0.52 and 0.98 for eyes operated with brilliant blue $\mathrm{G}$ and ICG, respectively.

As a limitation to our methodology, we believe that the affinity of TB to cellular matter may result in less homogeneous staining of ERM than that of the ILM, sometimes resulting in a reticular pattern that may, in fact, facilitate recognition in a way not represented by 
average contrast scores based on the entire ROIs. A slightly longer retinal exposure time and cooler TB baseline temperature favoring sinking of the substance onto the retina may be other factors that may have favored TB performance in relation to BBG.

All BBG was from batches made before the addition of deuterium, which has been recently added to the formula by the manufacturer to promote descent of the solution within the vitreous cavity and, thus, contact between the dye and the posterior pole ILM. In fact, first clinical reports observed improved staining of the ILM with this modified BBG. ${ }^{51}$

An important restriction has been that the classification of ERM versus ILM was based exclusively on the characterization of video material and that histologic confirmation was not available because of the retrospective nature of this study. We believe, however, that postoperative review of the videos with the surgeon guaranteed maximum clinical accuracy and have demonstrated previously that visual distinction of ERM and ILM based on staining comportment is possible with a high degree of precision. ${ }^{46}$

There are a range of chromovitrectomy protocols currently in use, and our results are based on findings from the specific methodology of this study. Different protocols might generate divergent findings:

Trypan blue was injected under BSS and removed after 10 seconds. Application under BSS is recommended by some groups in the literature, ${ }^{52}$ but advocates of an application under air can also be found..$^{8,16,53}$ The storage at $4^{\circ} \mathrm{C}$ is intended to improve TB descent within the vitreous cavity. Brilliant blue $\mathrm{G}$ washout, however, was performed immediately in our patient sample, as recommended in the manufacturer's instruction leaflet, and BBG was conserved at room temperature according to the hospitals' protocols. As ICG is not approved for intravitreal use, explicit references for its usage are not obtainable. For the current patient sample, ICG strength and intravitreal retention time were chosen according to the clinical protocols of the participating study centers. Indocyanine green was applied under BSS at a concentration at the upper limit of the $0.05 \%$ to $1.25 \%$ range described in the literature, with or without fluid-air exchange. ${ }^{23,54}$ Published ICG washout times vary from immediate removal to 180 seconds, ${ }^{23,54,55}$ while 1 minute was used in our sample. The effect of variations in clinical application protocols on contrast strength has yet to be evaluated, but improved staining is expected with higher concentrations and extended exposure times based on preclinical findings. ${ }^{56}$ The distance of the tip of the needle from the macula during dye injection may also influence staining outcome. Although the injection needle was positioned in the central vitreous by all surgeons, an exact distance from the fovea was not specified in this retrospective study and may account for some degree of variability.

The bias chromaticity measurements may suffer based on the choice of the intraocular light source as has been discussed previously. ${ }^{14}$ All interventions in this study were carried out using the same mercury vapor-based endoillumination system, allowing comparison between subjects.

About the lens status, chromaticity scores may have been somewhat influenced by different light-filtering effects produced by the presence of a natural or an artificial lens. However, only 4 of 51 patients were phakic: 2 from the BBG group and 2 from the ICG group. All phakic patients presented average CIELAB score values within the 25 th to 75 th percentile so that the effect appears to be negligible, although statistical proof cannot be provided because of small sample size.

Future prospective trials with larger sample sizes should investigate whether variables such as patient age, lens status, dye concentrations, dye temperature, exposure time, distance of the injection needle from the fovea, application with or without fluid-air exchange, and choice of endoillumination influence staining performance of TB. Further research is also warranted to analyze whether BBG and TB staining properties are additive and, if so, whether the simultaneous application of both substances represents a useful technique to improve ILM staining at low dye concentrations.

\section{Conclusions}

Trypan blue represents a suitable vital dye for chromovitrectomy in general and for sequential macular surgery in particular. For chromovitrectomy, its approval status and ILM staining characteristics make it a worthwhile alternative to BBG. Trypan blue is highly valuable in sequential ERM and ILM peeling, as its additional affinity to ERM allows delineation of all the target tissues of this operation. Occasional indications of retinal toxicity need to be carefully monitored.

Key words: TB, trypan blue, BBG, brilliant blue G, chromovitrectomy, contrast perception, epiretinal membranes, ERM, internal limiting membrane, ILM.

\section{References}

1. Gandorfer A. Objective of pharmacologic vitreolysis. Dev Ophthalmol 2009;44:1-6.

2. Kelly NE, Wendel RT. Vitreous surgery for idiopathic macular holes. Results of a pilot study. Arch Ophthalmol 1991;109: 654-659. 
3. Trese MT, Chandler DB, Machemer R. Macular pucker. I. Prognostic criteria. Graefes Arch Clin Exp Ophthalmol 1983; 221:12-15

4. Gandorfer A, Messmer EM, Ulbig MW, Kampik A. Resolution of diabetic macular edema after surgical removal of the posterior hyaloid and the inner limiting membrane. Retina 2000;20: 126-133.

5. Brooks HL Jr. Macular hole surgery with and without internal limiting membrane peeling. Ophthalmology 2000;107:1939 1948; discussion 1948-1949.

6. Foulquier S, Glacet-Bernard A, Sterkers M, et al. Study of internal limiting membrane peeling in stage-3 and -4 idiopathic macular hole surgery [in French]. J Fr Ophtalmol 2002;25:1026-1031.

7. Sheidow TG, Blinder KJ, Holekamp N, et al. Outcome results in macular hole surgery: an evaluation of internal limiting membrane peeling with and without indocyanine green. Ophthalmology 2003;110:1697-1701.

8. Christensen UC, Kroyer K, Sander B, et al. Value of internal limiting membrane peeling in surgery for idiopathic macular hole stage 2 and 3: a randomised clinical trial. Br J Ophthalmol 2009;93:1005-1015.

9. Al-Abdulla NA, Thompson JT, Sjaarda RN. Results of macular hole surgery with and without epiretinal dissection or internal limiting membrane removal. Ophthalmology 2004;111:142-149.

10. Smiddy WE, Feuer W, Cordahi G. Internal limiting membrane peeling in macular hole surgery. Ophthalmology 2001;108: 1471-1476; discussion 1477-1478.

11. Haritoglou C, Gass CA, Schaumberger M, et al. Long-term follow-up after macular hole surgery with internal limiting membrane peeling. Am J Ophthalmol 2002;134:661-666.

12. Rodrigues EB, Meyer CH, Kroll P. Chromovitrectomy: a new field in vitreoretinal surgery. Graefes Arch Clin Exp Ophthalmol 2005;243:291-293.

13. Farah ME, Maia M, Furlani B, et al. Current concepts of trypan blue in chromovitrectomy. Dev Ophthalmol 2008;42:91-100.

14. Henrich PB, Priglinger SG, Haritoglou C, et al. Quantification of contrast recognizability during brilliant blue $\mathrm{G}$ (BBG) and indocyanine green (ICG) assisted chromovitrectomy. Invest Ophthalmol Vis Sci 2012;53:2561-2570.

15. Team RDC. A Language and Environment for Statistical Computing. Vienna, Austria: R Foundation for Statistical Computing, 2009

16. Mackenzie SE, Gandorfer A, Rohleder M, et al. Ultrastructure and retinal imaging of internal limiting membrane: a clinicopathologic correlation of trypan blue stain in macular hole surgery. Retina 2010;30:655-661.

17. Kadonosono K, Itoh N, Uchio E, et al. Staining of internal limiting membrane in macular hole surgery. Arch Ophthalmol 2000;118:1116-1118.

18. Gandorfer A, Messmer EM, Ulbig MW, Kampik A. Indocyanine green selectively stains the internal limiting membrane. Am J Ophthalmol 2001;131:387-388.

19. Henrich PB, Haritoglou C, Meyer P, et al. Anatomical and functional outcome in brilliant blue $\mathrm{G}$ assisted chromovitrectomy. Acta Ophthalmol 2010;88:588-593.

20. Gandorfer A, Haritoglou C, Gass CA, et al. Indocyanine greenassisted peeling of the internal limiting membrane may cause retinal damage. Am J Ophthalmol 2001;132:431-433.

21. Haritoglou C, Gandorfer A, Gass CA, et al. Indocyanine greenassisted peeling of the internal limiting membrane in macular hole surgery affects visual outcome: a clinicopathologic correlation. Am J Ophthalmol 2002;134:836-841.

22. Engelbrecht NE, Freeman J, Sternberg P Jr, et al. Retinal pigment epithelial changes after macular hole surgery with indocyanine green-assisted internal limiting membrane peeling. Am J Ophthalmol 2002;133:89-94.

23. Gass CA, Haritoglou C, Schaumberger M, Kampik A. Functional outcome of macular hole surgery with and without indocyanine green-assisted peeling of the internal limiting membrane. Graefes Arch Clin Exp Ophthalmol 2003;241:716-720.

24. Kwok AK, Lai TY, Man-Chan W, Woo DC. Indocyanine green assisted retinal internal limiting membrane removal in stage 3 or 4 macular hole surgery. Br J Ophthalmol 2003;87: 71-74.

25. Yuen D, Gonder J, Proulx A, et al. Comparison of the in vitro safety of intraocular dyes using two retinal cell lines: a focus on brilliant blue $\mathrm{G}$ and indocyanine green. Am J Ophthalmol 2009; 147:251-259.e252.

26. Awad D, Schrader I, Bartok M, et al. Comparative toxicology of trypan blue, brilliant blue $\mathrm{G}$, and their combination together with polyethylene glycol on human pigment epithelial cells. Invest Ophthalmol Vis Sci 2011;52:4085-4090.

27. Enaida H, Hisatomi T, Hata Y, et al. Brilliant blue G selectively stains the internal limiting membrane/brilliant blue G-assisted membrane peeling. Retina 2006;26:631-636.

28. Remy M, Thaler S, Schumann RG, et al. An in vivo evaluation of brilliant blue $\mathrm{G}$ in animals and humans. $\mathrm{Br} \mathrm{J}$ Ophthalmol 2008;92:1142-1147.

29. Melles GR, de Waard PW, Pameyer JH, Houdijn Beekhuis W. Trypan blue capsule staining to visualize the capsulorhexis in cataract surgery. J Cataract Refract Surg 1999;25:7-9.

30. Meyer CH, Rodrigues EB, Kroll P. Trypan blue has a high affinity to cellular structures such as epiretinal membrane. Am J Ophthalmol 2004;137:207-208; author reply 208.

31. Perrier M, Sebag M. Epiretinal membrane surgery assisted by trypan blue. Am J Ophthalmol 2003;135:909-911.

32. Teba FA, Mohr A, Eckardt C, et al. Trypan blue staining in vitreoretinal surgery. Ophthalmology 2003;110:2409-2412.

33. Perrier M, Sebag M. Trypan blue-assisted peeling of the internal limiting membrane during macular hole surgery. Am J Ophthalmol 2003;135:903-905.

34. Feron EJ, Veckeneer M, Parys-Van Ginderdeuren R, et al. Trypan blue staining of epiretinal membranes in proliferative vitreoretinopathy. Arch Ophthalmol 2002;120:141-144.

35. Li KK, Tang EW, Li PS, Wong D. Double peel using triamcinolone acetonide and trypan blue in the management of myopic macular hole with retinal detachment: a case-control study. Clin Experiment Ophthalmol 2010;38:664-668.

36. Li K, Wong D, Hiscott $\mathrm{P}$, et al. Trypan blue staining of internal limiting membrane and epiretinal membrane during vitrectomy: visual results and histopathological findings. $\mathrm{Br} \mathrm{J}$ Ophthalmol 2003;87:216-219.

37. Lee KL, Dean S, Guest S. A comparison of outcomes after indocyanine green and trypan blue assisted internal limiting membrane peeling during macular hole surgery. Br J Ophthalmol 2005;89:420-424.

38. Morales MC, Freire V, Asumendi A, et al. Comparative effects of six intraocular vital dyes on retinal pigment epithelial cells. Invest Ophthalmol Vis Sci 2010;51:6018-6029.

39. Schmid-Kubista KE, Lamar PD, Schenk A, et al. Comparison of macular function and visual fields after membrane blue or infracyanine green staining in vitreoretinal surgery. Graefes Arch Clin Exp Ophthalmol 2010;248:381-388.

40. Veckeneer M, van Overdam K, Monzer J, et al. Ocular toxicity study of trypan blue injected into the vitreous cavity of rabbit eyes. Graefes Arch Clin Exp Ophthalmol 2001;239:698-704.

41. Melles GR. A disagreement. Ophthalmology 2004;111:193; author reply 194-195. 
42. Haritoglou C, Gandorfer A, Schaumberger M, et al. Trypan blue in macular pucker surgery: an evaluation of histology and functional outcome. Retina 2004;24:582-590.

43. Figueroa MS, Rebolleda G, Noval S, Contreras I. Evaluation of trypan-blue toxicity in macular hole surgery with electroretinography [in Spanish]. Arch Soc Esp Oftalmol 2008;83: 659-664.

44. Shimada H, Nakashizuka H, Hattori T, et al. Double staining with brilliant blue $\mathrm{G}$ and double peeling for epiretinal membranes. Ophthalmology 2009;116:1370-1376.

45. Kwok A, Lai TY, Yuen KS. Epiretinal membrane surgery with or without internal limiting membrane peeling. Clin Experiment Ophthalmol 2005;33:379-385.

46. Schumann RG, Gandorfer A, Eibl KH, et al. Sequential epiretinal membrane removal with internal limiting membrane peeling in brilliant blue G-assisted macular surgery. Br J Ophthalmol 1369;94:1369-1372.

47. Rodrigues EB, Meyer CH, Schmidt JC, Kroll P. Trypan blue stains the epiretinal membrane but not the internal limiting membrane. Br J Ophthalmol 2003;87:1431-1432.

48. Langhals $\mathrm{H}$, Haritoglou C. Chemical and spectroscopic aspects of the application of dyes in vitreoretinal surgery [in German]. Ophthalmologe 2009;106:16-20.
49. Taylor SH, Thorp JM. Properties and biological behaviour of Coomassie blue. Br Heart J 1959;21:492-496.

50. Lang JH, Lasser EC. Spectrophotometric studies of the binding of trypan blue to bovine serum albumin. Biochemistry 1967;6: 2403-2409.

51. Haritoglou C, Schumann RG, Kampik A, Gandorfer A. Heavy brilliant blue $\mathrm{G}$ for internal limiting membrane staining. Retina 2011;31:405-407.

52. Kalvoda J, Duskova J, Kubena A, et al. Morphometry of surgically removed internal limiting membrane during vitrectomy in diabetic macular edema. Graefes Arch Clin Exp Ophthalmol 2009;247:1307-1314.

53. Schumann RG, Gandorfer A, Priglinger SG, et al. Vital dyes for macular surgery: a comparative electron microscopy study of the internal limiting membrane. Retina 2009;29:669-676.

54. Gandorfer A, Haritoglou C, Kampik A. Toxicity of indocyanine green in vitreoretinal surgery. Dev Ophthalmol 2008;42:69-81.

55. Kanda S, Uemura A, Yamashita T, et al. Visual field defects after intravitreous administration of indocyanine green in macular hole surgery. Arch Ophthalmol 2004;122:1447-1451.

56. Rodrigues EB, Penha FM, de Paula Fiod Costa E, et al. Ability of new vital dyes to stain intraocular membranes and tissues in ocular surgery. Am J Ophthalmol 2009;149:265-277. 